\title{
cDNA cloning and mRNA expression of cat and dog Cdkall
}

This article was published in the following Dove Press journal:

Veterinary Medicine: Research and Reports

10 August 2012

Number of times this article has been viewed

\section{Ichiro Yamamoto \\ Shingo Ishikawa \\ Li Gebin \\ Hiroshi Takemitsu \\ Megumi Fujiwara \\ Nobuko Mori \\ Yutaka Hatano \\ Tomoko Suzuki \\ Akihiro Mori \\ Nobuhiro Nakao \\ Koh Kawasumi \\ Toshinori Sako \\ Toshiro Arai \\ Laboratory of Veterinary \\ Biochemistry, Nippon Veterinary and \\ Life Science University, Tokyo, Japan}

Correspondence: Ichiro Yamamoto Laboratory of Veterinary Biochemistry, School of Veterinary Medicine, Nippon Veterinary and Life Science University,

I-7-I Kyonancho, Musashino,

Tokyo, Japan

Tel +8I4223|4|5|

Email ichiroy@nvlu.ac.jp
Abstract: The cyclin-dependent kinase 5 regulatory subunit-associated protein 1-like 1 (CDKAL1) gene encodes methylthiotransferase, and the gene contains risk variants for type 2 diabetes in humans. In this study, we performed complementary DNA cloning for Cdkall in the cat and dog and characterized the tissue expression profiles of its messenger RNA. Cat and dog Cdkall complementary DNA encoded 576 and 578 amino acids, showing very high sequence homology to mammalian CDKAL1 (>88.4\%). Real-time polymerase chain reaction analyses revealed that $C d k a l l$ messenger RNA is highly expressed in smooth muscle and that tissue distribution of Cdkall is similar in cats and dogs. Genotyping analysis of single-nucleotide polymorphism for cat $C d k a l 1$ revealed that obese cats had different tendencies from normal cats. These findings suggest that the cat and dog Cdkall gene is highly conserved among mammals and that cat Cdkall may be a candidate marker for genetic diagnosis of obesity.

Keywords: cat, dog, Cdkall, obese, cDNA cloning, Q-PCR

\section{Introduction}

Genome-wide association studies (GWAS) have identified many novel susceptibility genes for type 2 diabetes since $2007 .{ }^{1}$ The gene for cyclin-dependent kinase 5 regulatory subunit-associated protein 1-like $1(C D K A L 1)$ has been identified as homologous to the gene for CDK5 regulatory subunit-associated protein 1 (CDK5RAP1), an inhibitor of CDK5 activation. Single-nucleotide polymorphisms (SNPs) of CDKAL1 were correlated with body mass index in East Asian populations. ${ }^{2,3}$ GWAS also showed correlations between $C D K A L 1$ variants and important physiological functions. $C D K A L 1$ variants were associated with decreases in betacell glucose sensitivity, ${ }^{4,5}$ reductions in first-phase insulin secretion, ${ }^{6}$ and lower birth weight. ${ }^{7}$ CDKAL1 is not only correlated with obesity but is also a risk loci for Crohn's disease. ${ }^{8}$ Functional analysis recently identified that human CDKAL1 belongs to the e-MtaB subfamily of methylthiotransferase. ${ }^{9}$ CDKAL1 is the first mammalian methylthiotransferase identified that biosynthesizes 2-methylthio- $N^{6}$ threonylcarbamoyladenosine (ms2t6A) in tRNA (Lys) (UUU) and is required for accurate translation of AAA and AAG codons. ${ }^{10}$

Type 1 diabetes ( $>50 \%$ of diabetes cases) appears to be the most common form of diabetes in dogs, whereas type 2 diabetes prevails in cats $(80 \%-95 \%$ of diabetes cases). ${ }^{11}$ However, very little information is available about Cdkall in cats and dogs because the cDNA sequences, gene structure, and mRNA expression mechanisms have not been determined. Therefore, the aim of this study was to determine the cDNA sequence of Cdkall and examine its mRNA expression profiles in cats and dogs. 


\section{Materials and methods}

\section{Cdkall cDNA cloning in a cat and a dog}

Total RNA from tissues of a cat (3-year-old male) and a dog (2-year-old male) were purchased from Zyagen (San Diego, CA). The amount of RNA was measured by spectrophotometry. A cDNA library was prepared from total liver RNA using the SMARTer RACE cDNA Amplification Kit (Clontech, Mountain View, CA). We referred to the human CDKAL1 cDNA sequence (GenBank accession number NM_017774) and the cat genome DNA sequence (GenBank accession number ti:914360954 and ti:728752993) to design specific primers for cat Cdkall. We designed primers 1 and 2 to obtain the partial cat cDNA sequence (Table 1). The polymerase chain reaction (PCR fragment) was cloned and the cDNA sequence was determined using the ABI Prism 310 Genetic Analyzer (Applied Biosystems, Foster City, CA). Primer 3 was used for amplification of the $3^{\prime}$ end of the cat Cdkall cDNA sequence, and primer 4 was used for $5^{\prime}$ rapid amplification of cDNA ends (RACE-PCR). Following cDNA cloning of cat $C d k a l l$, we obtained a partial Cdkall cDNA sequence from the dog using primers 5 and 6. Primers 7 and 8 were used for $3^{\prime}$ and $5^{\prime}$ RACE-PCR.

\section{The mRNA expression profile of cat Cdkall in tissues}

Total RNA $(1 \mu \mathrm{g})$ was reverse-transcribed using the QuantiTect Reverse Transcription Kit (Qiagen, Hilden, Germany). The genomic DNA was removed by DNase treatment, and cDNA was synthesized. One $\mu \mathrm{L}$ of the cDNA product was subjected to quantitative PCR (Q-PCR) according to the user's instructions for the Real-Time PCR System 7300

Table I Sequences and kind of primers used for polymerase chain reaction

\begin{tabular}{lll}
\hline Primer & Kind & Sequence $\mathbf{( 5}^{\prime} \mathbf{- 3}^{\prime} \mathbf{)}$ \\
\hline $\mathrm{I}$ & Sense & CCTCCTGTGATTCTCTCCTGGACGAC \\
2 & Antisense & CCCACACAAGGAAAAGAGGA \\
3 & Sense & GCCTCTGCTCCAGCCACACTTTTTA \\
4 & Antisense & GTGCTATTGCTCGGTGGGCTGTTTT \\
5 & Sense & GTGGCGGGACGTACGTGTCA \\
6 & Antisense & AATTTGGATTCCTGAAAGACACATA \\
7 & Sense & TATGAAAGGGCAGCCAGTAT \\
8 & Antisense & GAGAACAACCCCATGTTCGCATCC \\
9 & Sense & CCAAGCCTCTTTATTAACCAGTT \\
10 & Antisense & AAGATTCTTCTGTCACTAACACTTG \\
11 & Sense & GCCAACCGTGAGAAGATGACT \\
12 & Antisense & CTGGTATTGTCATGGACTCTGGG \\
13 & Sense & ATGCTTTTCTGCACATACCG \\
14 & Antisense & GAAGGCAGTCAACGTCTGGT \\
15 & Sense & CGGTCCAGTCTGCCTCTG \\
\hline
\end{tabular}

(Applied Biosystems). PCR was performed at $95^{\circ} \mathrm{C}$ for 5 seconds and $60^{\circ} \mathrm{C}$ for 35 seconds in $20 \mu \mathrm{L}$ of buffer containing $1 \times$ Premix EX Taq II (Takara, Otsu, Japan), $1 \times$ ROX reference dye, and $0.4 \mu \mathrm{M}$ each of primers 9 and 10 for cat and dog Cdkall. Primers 11 and 12 derived from the cat and dog beta-actin cDNA sequence were used for beta-actin mRNA. Following real-time PCR, the fragment was subjected to dissociation-curve analysis to avoid nonspecific PCR amplification. Quantitative measurements were performed by establishing a linear amplification curve from serial dilutions of a plasmid containing dog Cdkall and beta-actin cDNA fragments.

\section{Detection of SNP in the cat genome}

Seventy-four client-owned cats ( 37 females and 37 males, 4-17 years old) from five veterinary clinics were used. All cats were subjected to medical examinations at their veterinary clinics between March 2008 and May 2011 and were not being clinically treated for any disease. Cats were assessed by a body condition score (BCS, 1-5) and thus divided as follows: normal healthy control and obese (BCS $>4$ ) cats. Genomic DNA was extracted from $100 \mu \mathrm{L}$ of whole blood from obese and normal cats using a DNA Extractor WB Kit (Wako, Osaka, Japan). The quantity of the extracted genomic DNA was measured by spectrophotometry. PCR was performed with $1 \times$ Ex Taq buffer and $0.2 \mu \mathrm{M}$ of primers 13 and 14. The amplified PCR products were cleaned up and sequenced using primer 15 .

\section{Statistical analysis}

Data are presented as mean \pm standard error of the mean and were analyzed using Tukey's test following analysis of variance. All analyses were performed using GraphPad Prism (GraphPad Software, San Diego, CA).

\section{Results and discussion}

We cloned the Cdkall cDNA from the cat and dog. The cat cDNA sequence consisted of a 160-bp $5^{\prime}$ untranslated region (UTR), a 1728-bp open reading frame (ORF), and a 953-bp 3' UTR. The dog Cdkall cDNA sequence consisted of a 128-bp 5' UTR, a 1734-bp ORF, and a 906-bp 3' UTR. The predicted cat and dog Cdkall amino acid sequences were compared with those of other animals, which revealed high sequence similarity (Figure 1) (cat vs dog [92.1\%], vs human [91.3\%], vs cow [91.1\%], vs mouse [88.4\%], vs frog [82.6\%], vs chicken [80.9\%], vs salmon [77.2\%]). The predicted amino acid sequences of cat and dog Cdkall showed several conserved domains, including UPF0004, radical 

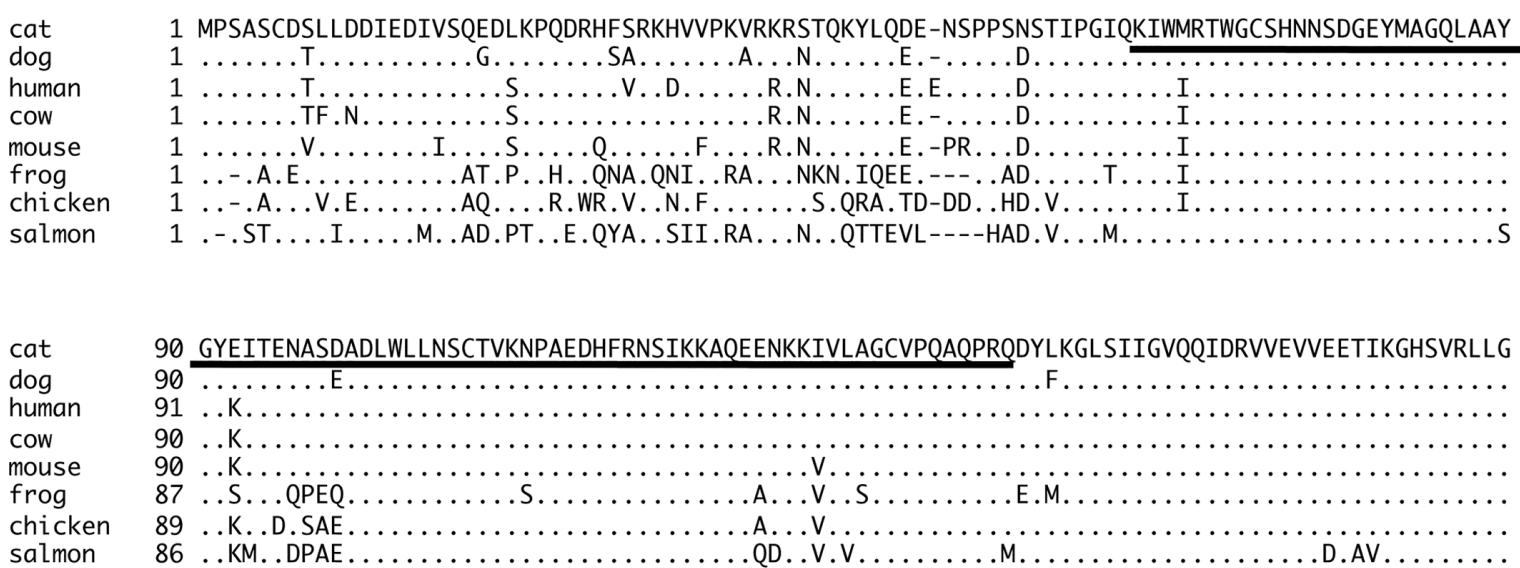

cat 180 QKKNNGKRLGGAPLDLPKIRKNPLIEIISINTGCLNACTYCKTKHARGNLASYPIDELVDRAKOSFO-EGVCEIWLTSEDTGAYGRDIGT

dog

Human

cow

mouse

frog

chicken

salmon

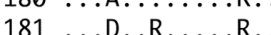

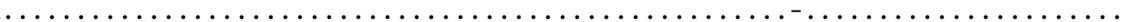

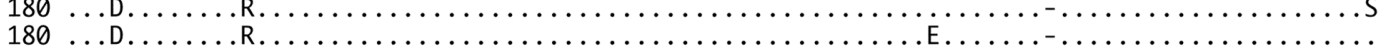

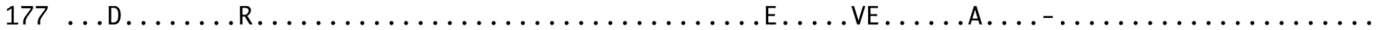

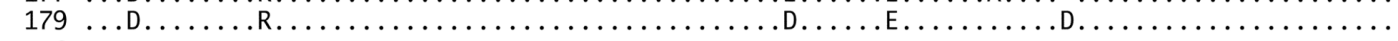

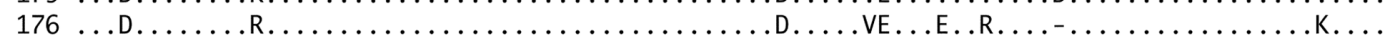

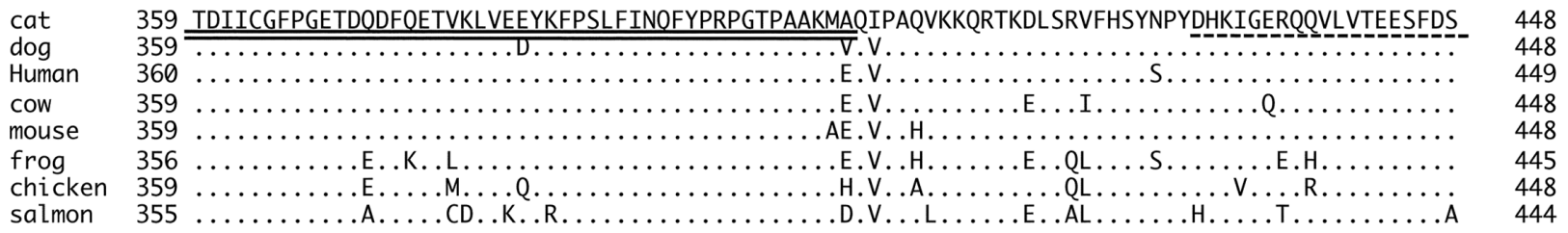

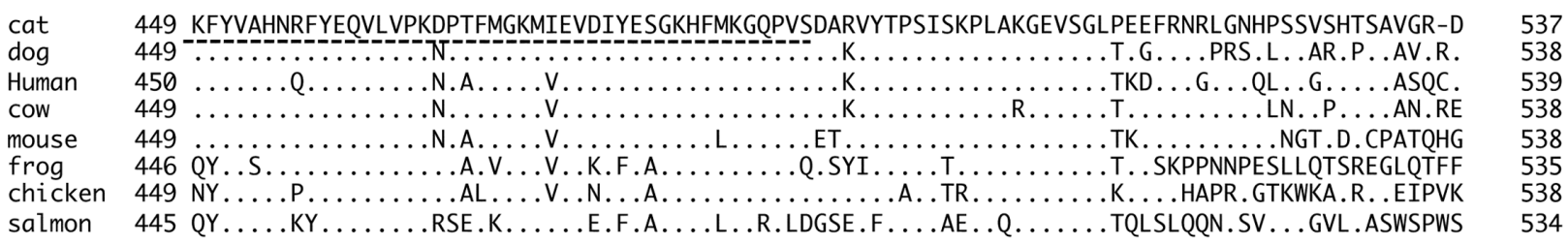

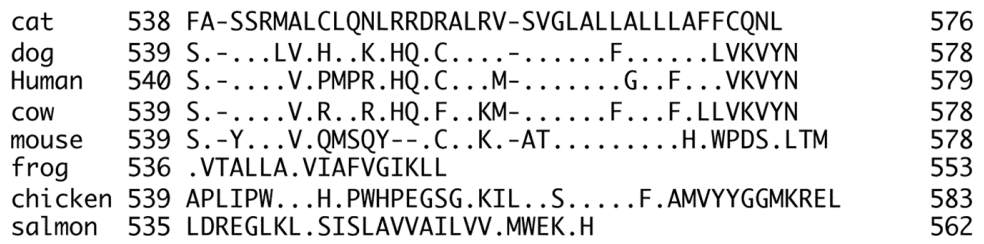

Figure I Comparison of the cyclin-dependent kinase 5 regulatory subunit-associated protein I-like I (CdkalI) amino acid sequence of cats with those of other animals. Notes: The amino acid sequence of cat Cdkall (AB720722) was aligned with those of dog Cdkall (AB720723), human CDKALI (NP_060244), cow Cdkall (NP_00I I79620), mouse Cdkall (NP_653II9), frog Cdkall (NP_989I94), chicken Cdkall (XP_4I89I4), and salmon Cdkall (NP_00II67I48). Dots matched to cat Cdkall. Single underline indicates UPF0004 domain, double underline indicates radical S-adenosylmethionine domain, and broken underline indicates tRNA-binding domain. 

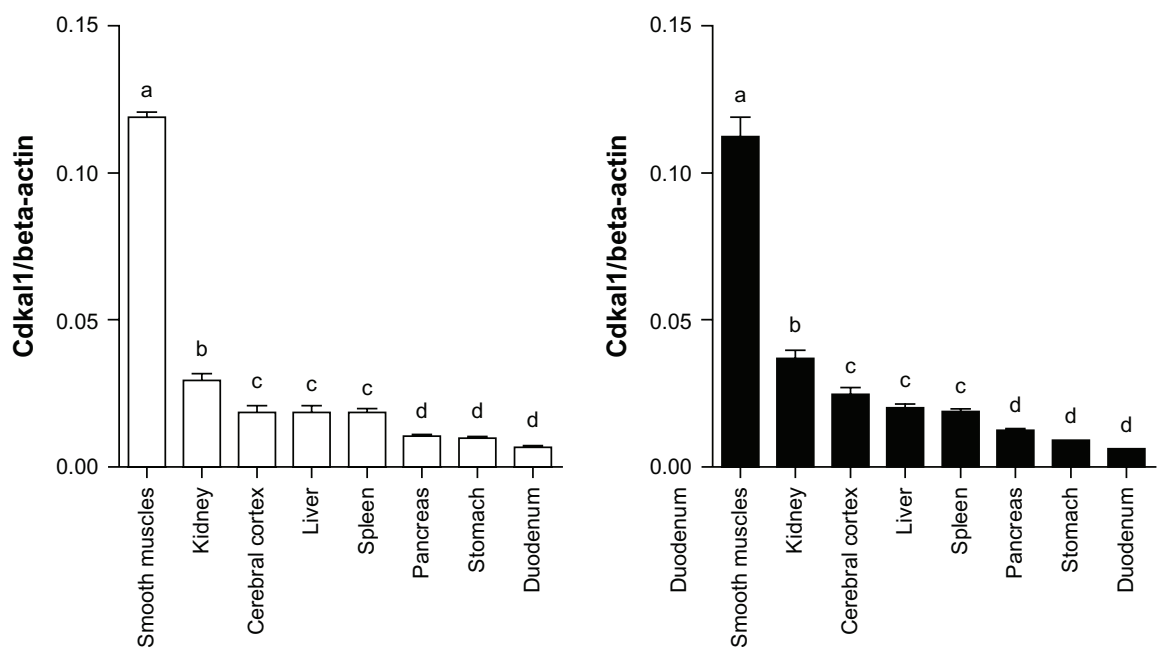

Figure 2 Tissue distribution profile of cyclin-dependent kinase 5 regulatory subunit-associated protein I-like I (Cdkall) mRNA in the dog (left; open box bars) and cat (right; closed box bars).

Notes: The expression levels of Cdkall mRNA in tissues of a 2-year-old male dog and a 3-year-old male cat were determined by real-time polymerase chain reaction. Each value of Cdkall mRNA was normalized to that of beta-actin mRNA and is the mean \pm standard error of mean ( $n=3$ ) of triplicate experiments for an individual RNA sample. The statistical analysis was performed using Tukey's test following analysis of variance. Values with different letters are significantly different $(P<0.05)$ in each dog and cat.

$S$-adenosylmethionine (SAM), and tRNA-binding (TRAM) domain. The UPF0004 domain has not been characterized structurally, but it contains three highly conserved cysteine residues. ${ }^{9}$ The radical SAM domain is a catalytic domain with suggested importance in tRNA modification, ${ }^{12}$ and the TRAM domain is involved in substrate (tRNA or protein) recognition..$^{9,13}$ Our findings suggest that the Cdkall gene may be functionally conserved in dogs and cats in the same manner as the human $C D K A L 1$ gene.

We determined the structure of the dog Cdkall gene using the cDNA sequence and the BLAST Genome database (http:// blast.ncbi.nlm.nih.gov). BLAST genome analysis revealed that the dog Cdkall gene consists of 15 exons and approximately 630-kb introns on chromosome 35. Human CDKAL1, which consists of 16 exons, is an approximately $700-\mathrm{kb}$ gene located on chromosome 6. Bovine Cdkall, which consists of 15 exons, is an approximately $600-\mathrm{kb}$ gene located on chromosome 23. The cat Cdkall gene consists of 16 exons and is approximately $730 \mathrm{~kb}$ in length. The discrepancies in the total exon numbers were caused by the differences in $5^{\prime}$ UTR. Although the start codon is located on exon 3 in humans and the cat, it is located on exon 2 in other animals.

We determined the Cdkall mRNA expression profiles by Q-PCR to examine the tissue-specific mRNA expression mechanism using cat and dog specific primers (Figure 2). The expression levels of cat and dog Cdkal1 mRNA were normalized to those of beta-actin mRNA. Cdkall mRNA was expressed in all examined tissues of a 3-year-old male cat and a 2-year-old male dog. The highest level of Cdkall
mRNA expression was observed in muscle, and it matched with the tissue distribution of human CDKAL1 mRNA. ${ }^{1}$ A comparison of the Cdkall expression profiles in cats and dogs showed a similar tendency, suggesting that the cat and dog Cdkall genes play a role in these tissues. The Cdkall mRNA expression profiles of dogs and cats also revealed the conservation of regulation of this expression.

We attempted to detect SNPs in obese cats on the basis of the Cdkall genomic structure because type 2 diabetes prevails in cats. ${ }^{11}$ The frequencies of the SNP genotypes in normal and obese cats are shown in Table 2 . The following two SNPs were observed: the T-allele frequency for rs44089532 of normal and obese cats was lower than the C-allele frequency, but the T-allele frequency for rs44089531 of normal cats was higher than the C-allele frequency and that of obese cats was equal to the $\mathrm{C}$-allele frequency. These two SNPs were located on intron 11 of the cat Cdkall gene. Many human GWAS have revealed four SNPs on intron 5 of the human CDKAL1 gene. ${ }^{14}$ Our findings suggest that rs44089531 may play an important rule

Table 2 Genotypes and allele frequencies in introns of $C d k a l l$ in normal and obese cat

\begin{tabular}{|c|c|c|c|c|c|}
\hline \multirow{2}{*}{$\begin{array}{l}\text { Genotype and allele } \\
\text { frequency }\end{array}$} & \multicolumn{3}{|c|}{ Genotype } & \multicolumn{2}{|c|}{ Allele frequency } \\
\hline & $\mathrm{C} / \mathrm{C}$ & $\mathbf{C} / \mathbf{T}$ & $\mathbf{T} / \mathbf{T}$ & C & $\mathbf{T}$ \\
\hline rs44089532 normal & 20 & 2 & I & 0.91 & 0.09 \\
\hline obese & 32 & 19 & 0 & 0.81 & 0.19 \\
\hline rs4408953I normal & 8 & 2 & 13 & 0.39 & 0.61 \\
\hline obese & II & 29 & II & 0.50 & 0.50 \\
\hline
\end{tabular}


in the pathogenesis of type 2 diabetes in cats. Because cats are thought to be good animal models of type 2 diabetes, functional SNP marker information will contribute to translational studies of humans and other animals. We did not complete SNP genotyping in cats, thus further analysis is required to determine whether SNPs found in this study would be helpful in diabetes diagnosis in obese cats.

\section{Acknowledgments}

This study was supported by a Grant-in-Aid for Young Scientists (B 22780270) and a Grant-in-Aid for Scientific Research (21380195 to Arai) from the Ministry of Education, Culture, Sports, Science and Technology, Japan.

These sequence data have been submitted to DDBJ/ EMBL/GenBank database under accession No AB720722 (cat Cdkal1) and AB720723 (dog Cdkal1).

\section{Disclosure}

The authors report no conflicts of interest in this work.

\section{References}

1. Zeggini E, Weedon MN, Lindgren CM, et al. Replication of genome-wide association signals in UK samples reveals risk loci for type 2 diabetes. Science. 2007;316:1336-1341.

2. Okada Y, Kubo M, Ohmiya H, et al. Common variants at CDKAL1 and KLF9 are associated with body mass index in east Asian populations. Nat Genet. 2012;44:302-306.

3. Wen W, Cho YS, Zheng W, et al. Meta-analysis identifies common variants associated with body mass index in east Asians. Nat Genet. 2012; 44:307-311.
4. Pascoe L, Tura A, Patel SK, et al. Common variants of the novel type 2 diabetes genes CDKAL1 and HHEX/IDE are associated with decreased pancreatic beta-cell function. Diabetes. 2007;56:3101-3104.

5. Pascoe L, Frayling TM, Weedon MN, et al. Beta cell glucose sensitivity is decreased by $39 \%$ in non-diabetic individuals carrying multiple diabetes-risk alleles compared with those with no risk alleles. Diabetologia. 2008;51:1989-1992.

6. Groenewoud MJ, Dekker JM, Fritsche A, et al. Variants of CDKAL1 and IGF2BP2 affect first-phase insulin secretion during hyperglycaemic clamps. Diabetologia. 2008;51:1659-1663.

7. Zhao J, Li M, Bradfield JP, Wang K, et al. Examination of type 2 diabetes loci implicates CDKAL1 as a birth weight gene. Diabetes. 2009;58:2414-2418.

8. Barrett JC, Hansoul S, Nicolae DL, et al. Genome-wide association defines more than 30 distinct susceptibility loci for Crohn's disease. Nat Genet. 2008;40:955-962.

9. Arragain S, Handelman SK, Forouhar F, et al. Identification of eukaryotic and prokaryotic methylthiotransferase for biosynthesis of 2-methylthio- $N^{6}$-threonylcarbamoyladenosine in tRNA. $J$ Biol Chem. 2010;285:28425-28433.

10. Wei FY, Suzuki T, Watanabe S, et al. Deficit of tRNA(Lys) modification by Cdkal1 causes the development of type 2 diabetes in mice. J Clin Invest. 2011;121:3598-3608.

11. Rand JS, Fleeman LM, Farrow HA, et al. Canine and feline diabetes mellitus: nature or nurture? J Nutr. 2004;134:2072S-2080S.

12. Wei FY, Tomizawa K. Functional loss of Cdkall, a novel tRNA modification enzyme, causes the development of type 2 diabetes. Endocr $J$. 2011;58(10):819-825.

13. Anantharaman V, Koonin EV, Aravind L. TRAM, a predicted RNAbinding domain, common to tRNA uracil methylation and adenine thiolation enzymes. FEMS Microbiol Lett. 2001;197:215-221.

14. Tomizawa K. Functions of Cdk5 in non-neuronal tissues: focusing on regulation of insulin secretion [Japanese]. Tanpakushitsu Kakusan Koso. 2009;54:808-812.
Veterinary Medicine: Research and Reports

\section{Publish your work in this journal}

Veterinary Medicine: Research and Reports is an international, peer-reviewed, open access journal publishing original research, case reports, editorials, reviews and commentaries on all areas of veterinary medicine. The manuscript management system is completely online and includes a very quick and fair peer-review system.

\section{Dovepress}

Visit http://www.dovepress.com/testimonials.php to read real quotes from published authors. 